\title{
COMPUTER-BASED SURGERY PLANNING FOR LIVING LIVER DONATION
}

\author{
H.-P. Meinzer ${ }^{\mathrm{a},{ }^{*}, \text { P. Schemmer }}{ }^{\mathrm{b}}$, M. Schöbinger ${ }^{\mathrm{a}}$, M. Nolden ${ }^{\mathrm{a}}$, T. Heimann ${ }^{\mathrm{a}}$, \\ B. Yalcin ${ }^{\mathrm{a}}$, G.M. Richter ${ }^{\mathrm{c}}$, T. Kraus ${ }^{\mathrm{b}}$, M.W. Büchler ${ }^{\mathrm{b}}$, M. Thorn ${ }^{\mathrm{a}}$ \\ ${ }^{a}$ Div. Medical and Biological Informatics, German Cancer Research Center, 69120 Heidelberg, Germany \\ ${ }^{\mathrm{b}}$ Dept. of Surgery, Ruprecht-Karls-University, 69120 Heidelberg, Germany \\ ${ }^{\mathrm{c}}$ Dept. of Radiology, Ruprecht-Karls-University,69120 Heidelberg, Germany
}

\section{Comission WG V/3}

KEYWORDS: Living donor live transplantation, operation planning, computer based planning, volumetry, anatomical evaluation

\begin{abstract}
:
The aim of this project is computer-based planning of a partial organ resection for three dimensional visualisation of anatomical structures and volumetric estimations. Using quantitative parameters for the estimation of the operation risk, the selection of patients can be further objectified. Another important effect of this project is support for surgical interventions by predefinition of different operation scenarios. All preoperative analysis results are based on tomographic images acquired during the clinical routine. In the end, the integration of planning results into the operation theater as well as into the clinical workflow is part of this project. Surgical strategy is determined by the exact location of the tumor, the respective donor liver part, and the proximity of supporting and draining vessels. Depending on the acquired images, the portal, hepatic, arterial and biliary systems may be analyzed. Therefore, fundamentals for the qualitative and quantitative analysis of images have been developed and implemented since the beginning of the project. The qualitative aspects are, e.g., the three dimensional visualisation of vessel structures, tumors, the security margin and the organ itself. These were evaluated for use in the clinical routine. Preoperatively, the operation strategy is planned by segmenting the important anatomical structures and defining the different vessel systems using a special operation planning software. The visualisation and volumetric results can be visualised and adapted during surgical intervention with the help of a beamer and a touchscreen monitor installed above the patient. In living donor liver transplantation (LDLT) the most important factor is to ensure that the anatomy of the donor patient allows surgical splitting of the liver. Also, a volumetric analysis of the donor liver is of interest to achieving less complicated surgery. Actually, all LDLTs performed in our Surgical Clinic are supported by this operation planning system.
\end{abstract}

\section{INTRODUCTION}

Live donor liver transplantation was first initiated in children in 1989 in response to the severe shortage of pediatric organ donors (Raia, 1989; Broelsch 1990). Improvements in surgical technique resulted in superior survival compared with cadaveric transplantations (Broelsch, 1991; Otte 1999). The expansion of live donor liver transplantation to the adult population initially began in countries where the availability of cadaveric donors was scarce and in some cases totally unavailable (Lo 1996a; Lo. 1996b; Kawasaki 1998). Severe shortages of cadaveric organs along with the exponential growth in the number of patients with cirrhosis secondary to the epidemic of hepatitis $\mathrm{C}$ have resulted in an increasing acceptance of live donor adult liver transplantation (LDLT) in Europe and the United States (Marcos, 2000a; Malago, 1999).

The donor selection is normally done in three phases (Pomfret 2001). In the first phase a potential donor is identified by obtaining demographic data and documenting the relationship between the donor and the recipient. After explanation of the surgical procedure and description of the evaluation process a laboratory estimation of blood compatibility and normal screening is done.

After passing the first phase the anatomical conditions of the donor are clarified in the second phase. For that reason CT images are acquired for the survey of the different vessel systems (portal vein, liver vein, bilary duct and arteries) within the donor liver. With the help of these images the feasibility and complexity of the surgical procedure is estimated. After the anatomical analysis a psychological estimation of the donor himself and his familiar setting is performed.

After the declarative statement of the laboratory, the radiology, internists, psychologists and ethical commission that the surgical intervention is possible for the donor the evaluation process gets into the third phase. In that last phase the recipient is re-evaluated to ensure that he has not become too sick for LDLT.

The process chain, especially the second phase, reflects one of the main problems in donor evaluation: the anatomical examination. The analysis of the vascular structures is done with the help of two-dimensional CT-images showing the vessels a highlighted dots. Only very experienced radiologists are able to verify the three dimensional structures of all vessels. A risk-aggravating factor is the fact that no images exist showing all different vessel-systems in one visualisation. Additionally a valid volumetric estimation of the donor graft size and the remaining liver tissue can't be done directly on the primary data.

To close that gap in donor evaluation we developed a computerbased operation-planning system, evaluated the volumetric estimation function and integrated this system in the clinical workflow.

\section{MATERIAL AND METHODS}

Different approaches have been made for the planning (Selle, 2000a) or the training and education (Marescaux, 1998) in this field of research. Using the computer-based operation planning system developed in Heidelberg it is possible to preoperatively 
and non-invasively analyse individual anatomy (Glombitza, 1998; Meinzer, 2002). The image data consists of contrasting agent enhanced CT images. Segmental classification is calculated by means of an analysis of portal vein structure. It defines volumes of the liver tissue components that are assigned to the different branches of the vessel tree.

The integration of computer-aided operation planning in this field requires that all steps of the analysis process are embedded in a framework that enables the reception of image data and forwarding of results. The individual procedural steps are:

- registration of the different CT-phases (venous, arterial, bilary),

- $\quad$ segmentation of liver and other organs to be visualised in the operation planning proposal. These organs are referred to as the anatomical landmarks.

- segmentation of the vessel system,

- differentiation of the vessel tree,

- calculation of a resection proposal and visualisation of the results,

- volumetric analysis of the graft size and the remaining liver,

- presentation during the preoperative meeting with radiologists, surgeons, internists, anesthetist,

- visualisation during the surgical intervention.

The system receives the image data with the aid of the CHILI ${ }^{\circledR}$ radiological system. CHILI is capable of communicating with the imaging device and stores all data in its own patient and image database. Furthermore, it encrypts forwarded and stored data sets.

\subsection{Registration of different CT-volumes}

Typically different images of the liver are acquired. Contrast agent is used to examine the anatomy of the four vessel systems - portal venous, hepatic, arterial and bile duct. For that reason images are taken in various points in time. As result three different CT-volumes are made which have to be fused to get a standardized coordinate system for all vessels. At the moment an affine approach is used which leads to very good correspondence (Böttger, 2003; Zitova, 2003).
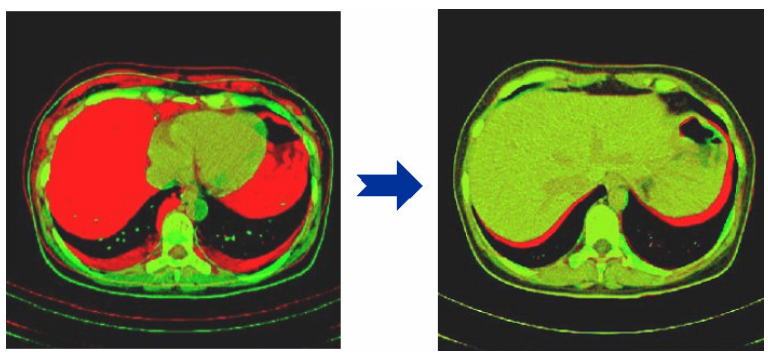

Registration of two CT- images of different phases (grey, light)

\subsection{Segmentation of the Anatomical Landmarks}

The next step is the segmentation of the image data in order to tag the liver. Other anatomical landmarks can also be identified for better orientation in the visualisation. Various manual and semi-automatic algorithms used for this purpose were integrated into the segmentation model and are stable for use in the clinical routine. This module provides basic interactions such as region growing, merge, cut, threshold, active contours, undo, propagation. The framework provides the opportunity for generating a graphical user interface for the image processing function. Therefore, new functions are easy to introduce. These algorithms can be used as two-dimensional tools to identify landmarks in each slice. To reduce the segmentation procedure time, many can also be applied to the whole volume (Kunert, 2004).

In order to facilitate the interaction between the end user and the segmentation module, a set of interaction patterns was incorporated and standard parameter values was used. Interaction patterns determine the ways in which the parameter values of an algorithm are obtained. For example, they can be obtained from the input image with a mouse click.

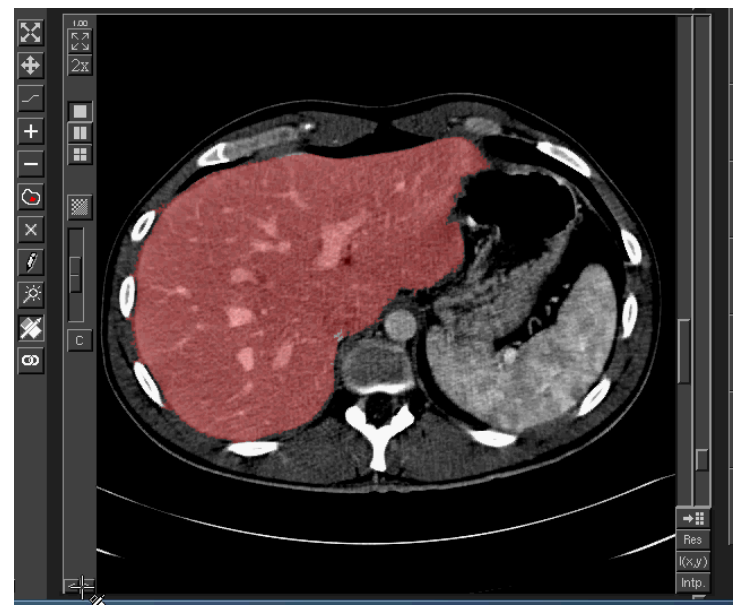

GUI of the segmentation tool

\subsection{Segmentation of the Vessel Tree}

A module divided in two parts performs vessel segmentation. The first one shows the input data and segmented landmarks. The user can interactively change the image's level/window values until only vessels are displayed. This sets a gray value. The portal vein system is used to calculate the resection strategy by selecting a starting point in the portal vein system's stem. The gray value and the starting point define the parameters of the second module part. A modified algorithm for vessel tree location according to Selle (Selle, 2000b) generates a symbolic description of the vessel tree (Schöbinger, 2002).

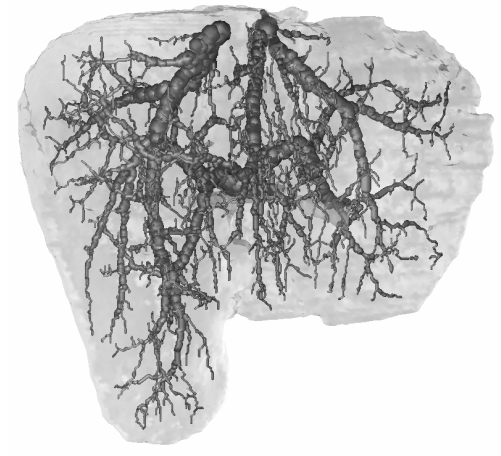

Visualisation of the liver and the venous system

The algorithm then passes through the entire data set searching for connected vessel structures. On the basis of a skeletonization a symbolic description is generated which includes the vessel-diameter at each position. A threedimensional reconstruction of the resulting vessel structure is shown, which the user can rotate and zoom to analyse the result. 
If the result does not correspond to the expected vein structure, the user can set another starting point or new range values.

The calculation of the resection strategy is currently based on the portal system structure but both parts of the venous system are enhanced. Because of noise and low resolution, pseudoconnections may occur between the portal and hepatic systems and, therefore, parts of the hepatic system may be included in the segmentation result. Usually, an editing step becomes necessary to separate portal from hepatic veins (Thorn, 2001a). The same extraction strategy takes place for the extraction of the arteries and bilary duct from the registered volume images.

\subsection{Separating vessel systems}

The main component of this module is the three-dimensional reconstruction created from the preceding module. In this module the user can edit the vessel system. The segmentation process may have generated invalid connections between the two venous systems but these can be severed interactively.

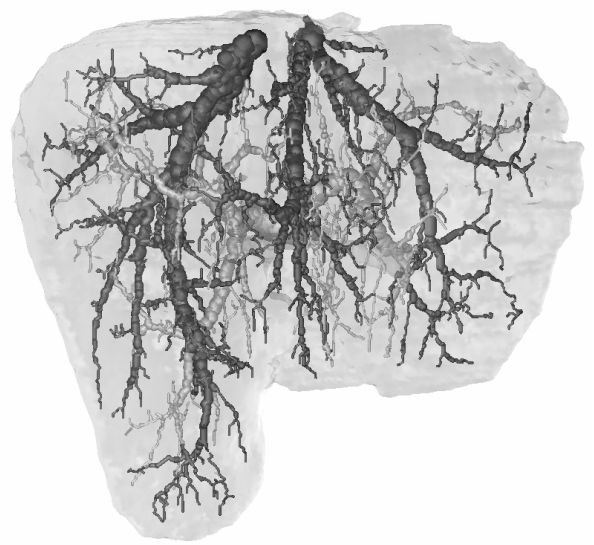

Visualisation of the liver and vessels after separation (portal vein in light-grey and hepatica in dark-grey)

The location of the invalid connections can be detected by calculating the path from the portal stem to a part of the hepatic system that belongs to the segmentation result. In this display the location where the segmentation result must be severed is easily detected. Each branch can be chosen interactively and tagged as a "stop branch". This tag severs the pseudoconnection. Only those parts of the vessel tree that are still connected to the portal stem will be brightly displayed. This is done until all parts of the hepatic system are removed from the segmentation result (Thorn, 2001a). Also the vessels that are represented in the other CT-phases - arterial and bile duct - are segmented and preprocessed in the same way.

\subsection{Resection Planning and Visualisation}

The next step of the planning procedure is the presentation of the results. This step includes a visualisation that shows the specific relation between the different vessel systems. Operation planning will visualise the proposed resection lines on the surface of the liver with the help of the OrgaNicer (Thorn, 2004a).

After segmentation and vessel extraction, vessel dependent tissue is calculated. To evaluate the volumetric results a study was performed that leads to an highly valid prognosis of the real liver volume (Thorn, 2004b). The quantitative results include total liver volume, graft size and remaining liver tissue.

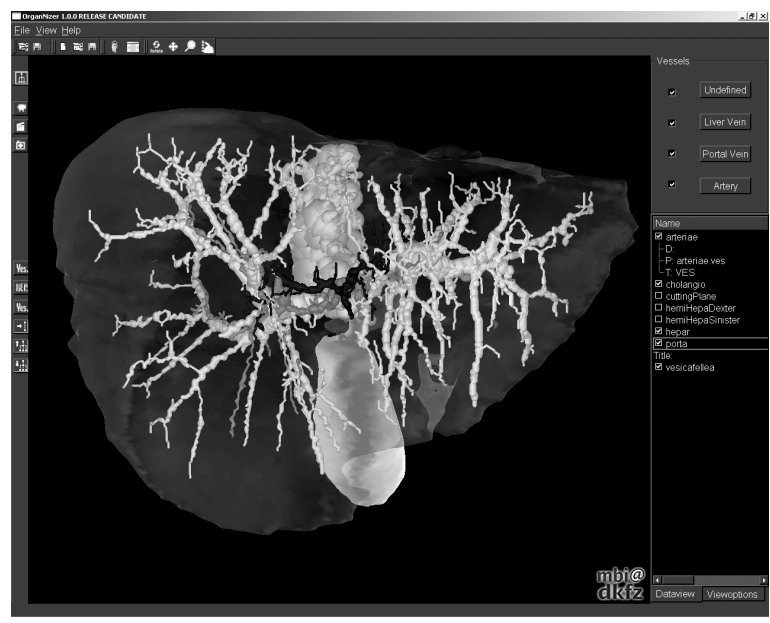

Screenshot of the OrgaNicer-tool showing a liver including the portal system (light-grey), the arteries (grey) and bile duct (dark-grey)

\subsection{Volumetric analysis}

In addition to the anatomical visualisation for the estimation of the complexity of the surgical intervention a volumetric examination is done. A crucial factor for a successful LDLT is the ratio between the liver mass and the body weight as well for the donor as for the recipient. As a rule the liver graft should be $1 \%$ of the body weight of the recipient and $0.8 \%$ of the body weight should be the remaining liver in the donor (Marcos, 2000b; Hess, 2002). For that reason the volume of the whole liver is calculated on the basis of the segmentation result (Thorn, 2004b). Afterwards the both liver halves are calculated and analysed in dependency of the portal vein structure.

\subsection{Intraoperative presentation}

The whole planning procedure take less than 1.5 hours. After the planning the results are presented during the preliminary discussion. In that meeting they are used to find the final decision if the potential donor will be able to spend one part of his liver. The volumetric results lead to a decision if the volume is sufficient for both patients. Afterwards the visualisation are examined concerning the feasibility of the surgical intervention.

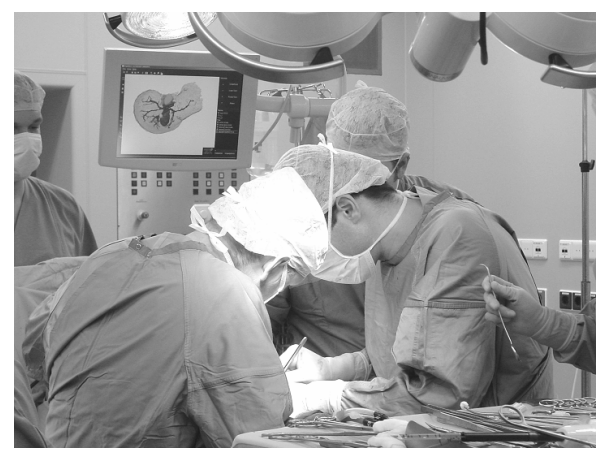

Presentation of the planning results in the operation room

Some days after the final decision is made the surgical intervention takes place. During the surgery the visualisations are presented with a monitor which is placed directly over the situs of the patient (Thorn, 2001b). The software can either be used with the help of a touch-screen monitor or by an additional 
assistant who is using a laptop. Mostly the second approach is used because the surgeon can keep his concentration to the surgical intervention. Mainly the visualisation helps the surgeon in the orientation during surgery. It also simplifies the discussion between the radiologist and the surgeon during the sonographic evaluation of the liver after mobilization.

\subsection{Distribution of the results}

For the easy exchange of the planning results between the German Cancer Research Center (DKFZ) and the UniversityClinic in Heidelberg a web-based system was established called LiverLine (Thorn, 2004c). This system provides the possibility of a secure distribution of the visualisation and volumetric estimation with the help of an virtual private network (Guichard, 2000; Yuan, 2001).

This system was established because there where the following drawbacks of the old workflow:

- the results of the operation planning were transferred by means of carrying a laptop (validation by the radiologist, presentation of the $3 \mathrm{D}$-visualisation for the surgeon in the steps). Therefore, the DKFZ coworker had to arrange an appointment, copy the data on a laptop and drive to the hospital.

- inconsistent distributed data storage by copying the data from the central computer to a laptop.

- the current state of the planning process was not traceable by the clinical partners during the planning phase.

- no documentation of the operation planning has taken place above.

These problems are solved by the web-based information system LiverLine as shown in the new workflow. All data of the liver operation planning is available by means of a web server and a database. The information from the operation planning is stored in the LiverLine database and can be accessed with a web browser. All partners involved have online access to the data of the operation planning so that they are not any longer temporal and spatially bound. At any time, the current status of the operation planning, as well as the original medical images and derived data (segmentations and 3D-visualisations) can be queried at any time from within the German Cancer Research Center and the clinic.

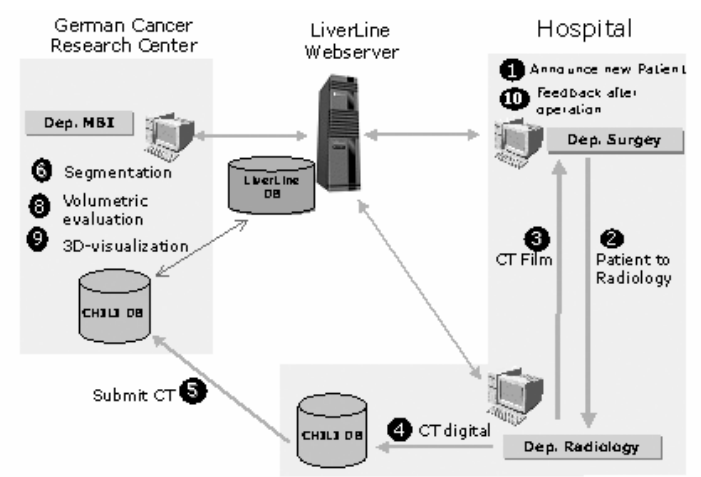

Optimized workflow with the help of the LiverLine

For the representation of the 3D-visualisation over the Internet the Virtual Reality Modelling Language (VRML) is used. The VRML model can be rotated and zoomed so the surgeon can view the liver from all sides.

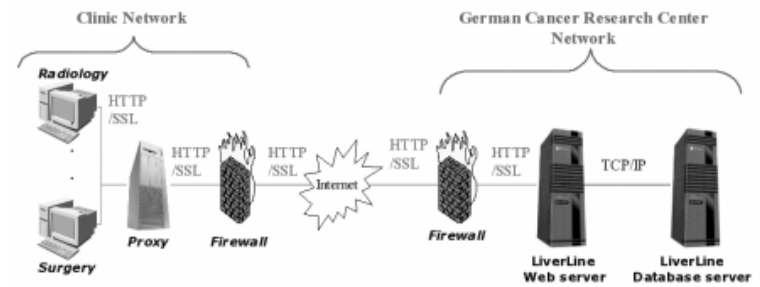

Security concept of the LiverLine

The safety requirements of medical information systems have highest priority. Therefore it must be guaranteed for the LiverLine that no unauthorized access to confidential patient data is possible. The most important features of such a internet solution are:

- Web server: authentication and access control

- Virtual Private Network (VPN) between the German Cancer Research Center and the hospital

- SSL (Secure Socket Layer) encryption for the security of the data communication

\section{RESULTS AND DISCUSSION}

The described planning procedure is completely integrated into the clinical workflow. For living donor liver transplantation it is a standardized method for donor selection and is performed for each donor in the Heidelberg University Clinic. It turned out that about $30 \%$ of planned potential donors were dismissed because of anatomical reasons. Also volumetric findings lead to disaffirmations of donors.

The presentation of the planning results topped the communication between all integrated medical disciplines. Especially the radiologists were easily able to point up their findings in the primary CT-images with the help of the threedimensional visualisations. Additionally during the surgical intervention the sonographic findings were traced with the help of the planning results.

With the help of the LiverLine the planning workflow was optimized. The clinicians are now able to analyze the findings of the computer-based planning without any longer temporal and spatially bounds. This leads to a transparent workflow that arises the acceptance of the planning procedure in the clinic. The next step will be the expansation of the planning service to other clinics which can easily be adapted with the LiverLine.

Our experiences in computer-based operation planning of the last six years, show a high acceptance and need of threedimensional reconstructions in the clinic. But there won't be any time of the physicians for the most time consuming work the segmentation procedure. For that reason we prefer to centralize this image processing step to be done in a competence center as a service provided to the clinicians in the future. With this work we illustrated how such a service can be provided.

\section{REFERENCES}

Broelsch CE, Emond JC, Whitington PF, Thistlethwaite JR, Baker AL, Lichtor JL., 1990, Application of reduced-size liver transplants as split grafts, auxiliary orthotopic grafts, and living related segmental transplants. Ann Surg. ;212:368-375. Discussion 375-377. 
Broelsch CE, Whitington PF, Emond JC, et al., 1991, Liver transplantation in children from living related donors: surgical techniques and results. Ann Surg;214:428-439.

Böttger T, Ruiter NV, Stotzka R, Bendl R, Herfarth KK. , 2003, Registration of CT and MRI Volume Data of the Liver. Lemke HU, VannierMW, Inamura K, Farman AG, Doi K, Reiber JHC (eds). CARS 2003. Computer Assisted Radiology and Surgery. Proceedings of the 17th International Congress and Exhibition. Amsterdam: Elsevier.

Glombitza G, Lamade W, Demiris AM, Göpfert MR, Mayer A, Bahner ML, Meinzer HP, Richter G, Lehner Th, Herfarth C., 1999, Virtual planning of liver resections: image processing, visualisation and volumetric evaluation. Int. Journal of Medical Informatics; 53 (2-3):225-237.

Guichard J., Pepelnjak I., 2000, MPLS and VPN Architecture: A Practical Guide to Understanding, Designing and Deploying MPLS and MPLS-Enabled VPNs, Cisco Press.

Hess D., Humar A., Sielaff T.D., 2002, Living related liver transplantation of recurrent hepatocellular carcinoma in normal liver. Clin Transpl; 16:240-242.

Kawasaki S, Makuuchi M, Matsunami H, et al., .1998, Living related liver transplantation in adults. Ann Surg;227:269-274.

Kunert T, Heimann T, Schröter A, Schöbinger M, Böttger T, Thorn M, Wolf I, Engelmann U, Meinzer HP, 2004, An Interactive System for Volume Segmentation in ComputerAssisted Surgery. Proceedings SPIE Conference Medical Imaging 2004; in press.

Lo CM, Chan KL, Fan ST, et al., 1996a, Living donor liver transplantation: the Hong Kong experience. Transplant Proc.;28:2390-2392.

Lo CM, Fan ST, Chan JK, Wei W, Lo RJ, Lai CL., 1996b, Minimum graft volume for successful adult-to-adult living donor liver transplantation for fulminant hepatic failure. Transplantation;62:696-698.

Malago M, Burdelski M, Broelsch CE., 1999, Present and future challenges in living related liver transplantation. Transplant Proc.;31:1777-1781.

Marcos A, Ham JM, Fisher RA, Olzinski AT, Posner MP. , 2000a, Single-center analysis of the first 40 adult-to-adult living donor liver transplants using the right lobe. Liver Transpl.;6:296-301.

Marcos, Fischer RA, Ham JM, 2000b, Liver regeneration and function in donor and recipient after right lobe adult to adult living donor liver transplantation. Transplantation;69:13751379 .

Marescaux J, Clement J-M, Tassetti V, et al., 1998, Virtual reality applied to hepatic surgery simulation: The next revolution. Ann Surg; 228 (5):627 - 637.

Meinzer HP, Thorn M, Cardenas, C., 2002, Computerized planning of liver surgery - an overview. Computers \& Graphics; 26/4: 569-576.

Otte JB, Reding R, de Ville de Goyet J., et al, 1999, Experience with living related liver transplantation in 63 children. Acta Gastroenterol Belg.;62:355-362.
Pomfret E., Polposelli J, Lewis W. et al., 2001, Live donor adult transplantation using right lobe grafts: donor evaluation and surgical outcome. Archives of Surgery; 136(4):425-433.

Raia S, Nery JR, Mies S., 1989, Liver transplantation from live donors [letter]. Lancet.;2:497.

Schöbinger M., 2002, Analyse von Gefäßstrukturen und versorgtem Gewebe, Diplomarbeit, Universität Heidelberg/ Fachhochschule Heilbronn, Heidelberg.

Selle D, Spindler W, 2000a, Schenk A. Computerized models minimize surgical risk. Diagnostic Imaging Europe; 16 (9):1620.

Selle D., 2000b, Analyse von Gefäßstrukturen in medizinischen Schichtdatensätzen für die computergestützte Operationsplanung, Aachen, Universität Bremen, Diss.

Thorn M, Vetter M, Cardenas C, Hassenpflug P, Fischer L, Grenacher L, Richter GM, Lamade W, Meinzer HP., 2001a, Interaktives Trennen von Gefäßbäumen am Beispiel der Leber. In Lehmann $\mathrm{T}$. et al. (Eds). Informatik Aktuell Bildverarbeitung für die Medizin 2001 - Algorithmen, Systeme, Anwendungen. Heidelberg: Springer; 147-151.

Thorn M, Fischer L, Cárdenas C, Vetter M, Hassenpflug P, Grenacher L, Richter GM, Lamadé W, Meinzer HP., 2001b, Integration of operation-planning results for liver surgery into the operation theatre. In Kim MH, Meinzer HP (eds). Proceedings of the Fifth Korea-Germany Joint Workshop on Advanced Medical Image Processing. Seoul, Korea, May 15th/16th, 2001. Seoul: Ewha Womans University.

Thorn M, Heimann T, Sonek C, Schöbinger M, Schemmer P, Grenacher L, Richter GM, Kraus T, Büchler MW, Meinzer HP.., 2004a, OrgaNicer - Eine Visualisierungssoftware für die Operationsplanung in der Leberchirurgie. In: Spath, D.; Haasis, K. (Hrsg.): Aktuelle Trends in der Softwareforschung Tagungsband zum doIT Software-Forschungstag 2003, IRB Verlag Stuttgart 2004, ISBN 3-8167-6453-3, S. 175-185

Thorn M, Kremer M, Heimann T, Schmied BM, Schemmer P, Richter GM, Z'graggen K, Büchler MW, Meinzer HP, 2004b, Accurate volume measurement in liver surgery - in vivo evaluation with a pig model. International Congress Series, CARS 2004; 1268C:730-734.

Thorn M, Yalcin B, Schemmer P, Grenacher L, Kraus T, Büchler MW, Meinzer HP., 2004c, LiverLine - ein webbasiertes Informationssystem für die computergestützte Leberoperationsplanung. In Tolxdorff T, Braun J, Handels H, Horsch A, Meinzer HP (eds). Bildverarbeitung für die Medizin 2004. Berlin: Springer (2004); 328-332.

Yuan R., Strayer W.T., 2001, Virtual Private Networks: Technologies and Solutions, Addison-Wesley Verlag.

Zitova B., Flusser J., 2003, Image registration methods: a survey. Image and Vision Computing; 21:977-1000.

\section{ACKNOWLEDGEMENT}

This is funded by the Tumorzentrum Heidelberg/Mannheim. 